The possibility of this force being electrostatic in origin is worth considering. At larger separations, of course, the net force due to the ions is repulsive, and the treatment of Langmuir ${ }^{7}$ essentially applies. But here we are dealing with the case where not only the Gouy, but also the Stern layers may overlap, and the essential assumption underlying the application of the Poisson-Boltzmann equation, that each ion moves in the mean potential field of all the ions, may break down. The ions will then rearrange themselves in a single layer, so that each one occupies a certain 'cell', bounded on either side by the two interacting surfaces, from which other ions are excluded. The force between the sheets will then be attractive, and its potential will increase uniformly with separation, at a rate depending on the dielectric constant of the medium. Assuming this is the same as for solid methanol, we get curve III in Fig. 2, and combining it with the curve of adsorption free energy we get curve IV, showing a stable position with two layers of methanol molecules between the montmorillonite sheets, which is what is actually found.

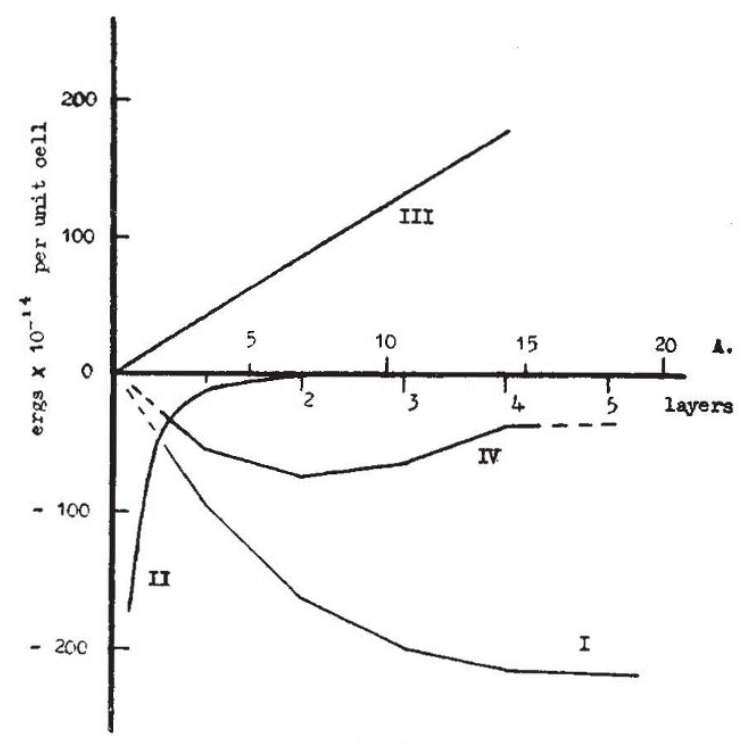

Fig. 2

The electrostatic force, unlike the van der Waals' force, is thus of the right order of magnitude to explain the observed effect. Its actual value is subject to error, because no account has been taken of the effect of segregation of the cations towards one or other of the two sheets. With 'bare' cations, this effect should lead to a considerable lowering of the attractive force at $10 \mathrm{~A}$. separation. It is probable, however, that the cations will co-ordinate polar groups (or residual water molecules) in such a way as to increase their effective size, and make this effect less marked. At separations greater than about $10 \mathrm{~A}$., such segregation is bound to set in, reducing the attractive force, until finally, with the attainment of the normal Gouy layer distribution, the force becomes repulsive. Actual values for the separation in organic complexes ${ }^{4}$ vary up to about $11 \mathrm{~A}$. The case of adsorption of large complex ions, for example as studied by Gieseking ${ }^{11}$, is, of course, quite a different one.

I am grateful to Dr. D. H. Bangham, Dr. S. Levine and Dr. R. K. Schofield for conversations on the subject of this communication, but they are not responsible for the opinions expressed in it.

${ }^{1}$ Bradley, W. F., J. Amer. Chem. Soc., 67, 975 (1945).

${ }^{2}$ Mac Ewan, D. M. C., Nature, 154, 577 (1944).

'MacEwan, D. M. C., Trans. Farad. Soc., 42, B, 226 (1946).

MacEwan, D. M. C., Trans. Farad. Soc., 44, 349 (1948).

'Bangham, D. H., and Mosallam, S., Proc. Roy. Soc., A, 166, 558 (1938). "Verwey, E. J. W., and Overbeek, J. Th. G., "Theory of the Stability
of Lyophobic Colloids" (Elsevier, 1948),

'Langmuir, I., J. Chem. Phys., 6, 873 (1938), in particular p. 893.

${ }^{8}$ Ref. 6 , p. 182.

Hofmann, U., Endell, K., and Wilm, D., Z. Krist., 86, 340 (1933).

${ }^{10}$ Ref. 6, p. 101 .

${ }^{11}$ Gieseking, J. E., Soil Sci., 47, 1 (1939); Ensminger, L. E., and Gieseking, J. E., ibid., 48, 467 (1939).

\section{HIGH VACUA GLENEAGLES CONVENTION}

$\mathrm{O}^{\mathrm{N}}$ $\mathrm{N}$ October 12 and 13 , the Society of Chemical Industry sponsored a convention on high vacua under the presidency of Sir Charles Darwin, director of the National Physical Laboratory. It is worthy of record that there was much appreciative comment at the choice of Gleneagles for the meeting, and the view was expressed widely that the so-called 'country house party' system in which interested persons meet in conditions of intimacy for a time with little or no distractions has much to recommend it.

The meetings opened with a brief historical survey and some personal recollections by Sir Charles Darwin. His remarks on the interaction of pure science and technological practice reminded his listeners that modern developments have produced a situation in which the contribution of the highly expert technician is now an essential to success. As he rightly remarked, it is no longer practicable for the physicist or the chemist in his research laboratory himself to make the apparatus he requires for his own use.

Dr. Saul Dushman, of Schenectady, dealt with the scientific aspects of vacuum technique. He referred to the advances resultant on the advent of the vacuum tungsten lamp and its developments, the magnetron, and fluorescent-screen cathode-ray tubes. The introduction of high-vacuum technique has enabled the production of high-energy accelerators such as the cyclotron, betatron and synchrotron. Many of the developments, the result of war-time demands, would astonish those concerned with these matters even ten years ago, he remarked, and it is fair to say that the technique has now emerged from the experimental stage to take its place as a valuable tool for the physicist. Oil-vapour pumps are available with speeds of exhaust ranging up to, and above, 7,000 litres/sec. (15,000 cu. ft./min.) and producing ultimate vacus ranging from $10^{-2}$ microns to $10^{-5}$ microns mercury pressure.

Dr. Dushman spoke of the difficulties of producing accurate gauges for such measurements; he believes that, in some cases at least, the limit of measurement has, for the time being, been reached.

Dr. J. C. Swallow and Dr. J. S. Gourlay, of Welwyn, discussed the applications of high vacua in the paint and plastics industries. The first part of their paper dealt with the technique of metallization now becoming increasingly important on a large scale. This technique involves the deposition in vacua of a thin partially transparent layer of metal over a surface. 


\section{No. 4128 December 11, $1948 \quad$ N A T U RE}

The metal is evaporated from a small source so placed that it impinges on the surface at an oblique angle. Irregularities on the surface produce local variations in the thickness of the metal layer, and the shadows cast by these enable their heights to be calculated. A very interesting process has also been developed for coating polymethyl methacrylate with aluminium. This is used to make the spherical plastic mirrors of diameters from 3 in. to 11 in. used in television projection.

In high-vacuum distillation it is hoped that the progress recently made will soon permit the separation of the various molecular species in a pure state. In plastics, short-path distillation can now be employed in the manufacture of a high molecular weight plasticizer by removing the ester from the reaction before its completion, which would require such high temperatures as to cause discoloration. In general, in a reaction $A+B=C+D$, the procedure is to remove by high-vacuum distillation $C$ or $D$, and the technical requirement of purer and still purer plasticizers of high molecular weight may well lead to the adoption of this procedure.

An interesting suggestion by Dr. Swallow was to consider not only the use of high-vacuum technique in the production of plastics, but also the use of plastics in high-vacuum apparatus, particularly for gaskets, inspection windows, and sheathing.

Dr. R. S. Morse, of Cambridge, Mass., contributed two papers on high-vacuum dehydration and distillation. An exciting feature of his papers was the evidence of applying highly complex processes to the production of very large quantities of materials like penicillin, sera, and the like. The cold-trap vapour pumping system in which air pressure is reduced to less than 50 microns of mercury with a mechanical pump is now in use on a large scale for drying biological preparations. For the production of penicillin and streptomycin, however, diffusion pumps are used to reduce the free-air pressure to 1-10 microns. The vapour is condensed and continuously removed as ice by rotating scraper blades. In an effort to eliminate all mechanical problems and convert a desiccant system to a continuous operation, continuous liquid chemical absorption processes are being studied. In these, free air is removed to $10-100$ microns and vapour is continuously absorbed with a hygroscopic medium. The medium is afterwards reconcentrated and, at reduced temperature, again circulated and additional water vapour removed. There are particular and unique problems involved in designing high-vacuum drier equipment, such as the heat transfer to the product. Efforts have been made also to reduce the drying time to a minimum, and Dr. Morse suggested that the time is approaching when high-vacuum drying operations will compete with more usual procedures such as spray drying.

Dr. E. W. M. Fawcett, of the Paint Research Station, discussed the application of high-vacuum distillation to the processing of triglyceride oils. Molecular distillation is limited generally to distillands in the molecular weight range $300-1,200$; it has a poor fractionating power and is rather high in cost. The rapid development of solvent fractionation processes may well also limit the application of the distillation process in some directions. It would appear, said Dr. Fawcett, that molecular distillation is a process for the special case, such as the recovery of vitamin A from fish liver oils, and, in his view, it is unlikely to be applied generally on a commercial scale. In contradistinction to its rather limited scope in commercial-scale practice, however, molecular distillation as a laboratory process has great possibilities and will undoubtedly find increasing usage with the perfection of the necessary high-vacuum technique.

Mr. R. A. Stauffer, of Cambridge, Mass., spoke on vacuum metallurgy, where much of the interest is still in the research and development stage. The two major advantages of processing metals in vacuo are that metals so processed are protected from reaction with gases, and those metallurgical reactions in which gaseous products are formed from solid or liquid reactants are favoured by maintaining a low gas pressure over the reactants. During the War very large quantities of magnesium were produced in Canada and the United States using a high-vacuum technique, but most of the plants are now closed because of high operating costs and poor plant locations. It is probable, said Mr. Stauffer, that lithium metal will be produced in the near future using vacuum techniques, and the production of other metals of the alkali and alkaline earth metal group have been studied, but so far only on a small scale. Small quantities of barium and cæsium are being made but the amount is limited to a few tons per year.

It is theoretically possible to drive reactions involving dissociation of metallic compounds which give rise to gaseous by-products; but in practice the number of metals which might be prepared by those techniques is extremely limited. Many of the halides can be dissociated by heating to high temperatures, but these reactions cannot be readily driven by vacuum techniques since most of the halides are quite volatile themselves and are removed, along with the halogen formed, as a by-product. A promising application for vacuum vaporization is in conjunction with the 'Kroll' process whereby titanium chloride is reduced with magnesium, forming a mixture of titanium, magnesium, and magnesium chloride from which titanium must be recovered. A vacuumvaporization technique is used for removal of magnesium and magnesium chloride in order to avoid a leaching operation which would contaminate the titanium.

Vacuum technology as applied to the design and construction of vacuum furnaces is progressing rapidly and new techniques for melting are being developed. There appears, however, to be little to be gained in the mechanical properties of metals by vacuum heat-treating operations.

Mr. Stauffer concluded by saying that the advantages to be gained by the use of vacuum technology have not been fully evaluated and in some cases the equipment required for its industrial application to metallurgical processes is not fully developed.

The final session was devoted to two papers, one by Dr. C. R. Burch, of Bristol, who spoke of developments arising out of experiments on vacuum distillation, considered as an illustration of the impact of one technology on another; and the other was by Prof. M. L. E. Oliphant, of Birmingham, who spoke of high vacua in nuclear physics, mentioning in particular some of the practical difficulties of work in this field. Dr. Burch's talk was largely historical for, as he explained, he has not worked on the subject for some years.

Prof. R. V. Jones, of Aberdeen, in his concluding remarks, directed attention to some of the main points made by the speakers and in the discussions. The convention concluded with an expression of thanks to those who had made it possible. 